Ophthalmologe $2021 \cdot 118: 1208-1210$ https://doi.org/10.1007/s00347-021-01522-1 Angenommen: 11. Oktober 2021 Online publiziert: 3. November 2021 (c) Springer Medizin Verlag $\mathrm{GmbH}$, ein Teil von Springer Nature 2021

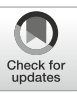

\section{Schlüsselstudien zum Glaukom der letzten 10 Jahre}

\author{
Karl Mercieca \\ Augenklinik, Universitätsklinikum Bonn, Bonn, Deutschland
}

Die englische Version dieses Beitrags ist unter https://doi.org/10.1007/s00347-021-01523-0 zu finden.

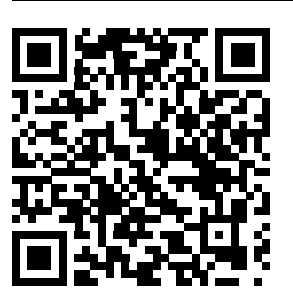

QR-Code scannen \& Beitrag online lesen
Die Möglichkeiten zur Diagnose und Behandlung des Glaukoms haben sich in den letzten 10 Jahren durch neue bildgebende Verfahren, pharmakologische Behandlungen und neuartige Operationsmethoden exponentiell erweitert. Aufgrund dieser umfangreichen Auswahl kann es ziemlich schwierig sein zu entscheiden, welche Option für jeden einzelnen Patienten am besten geeignet ist. Dies gilt umso mehr angesichts der umfangreichen Informationsflut, die von Herstellern, Forschern und Kollegen veröffentlicht und präsentiert wird. Mit diesem Leitthema haben wir uns daher entschieden, einige der bedeutenden wegweisenden Glaukomstudien, die in den letzten 10 Jahren veröffentlicht wurden, vorzustellen. Damit wollen wir die Anwendung evidenzbasierter Medizin bei der Planung von Untersuchungen und Behandlungen unserer Patienten fördern.

Das Glaukom ist eine der Hauptursachen für irreversiblen Sehverlust mit einer geschätzten weltweiten Prävalenz von 64,3 Mio. im Jahr 2013, die sich auf 76,0 Mio. im Jahr 2020 und 111,8 Mio. im Jahr 2040 erhöhen wird [1]. Da das Glaukom im Frühstadium hauptsächlich eine asymptomatische Erkrankung ist, sind die Identifizierung von Risikofaktoren und eine zeitnahe Diagnose entscheidend, um ein Fortschreiten der Krankheit und einen signifikanten Verlust des Sehvermögens zu vermeiden. Sobald die Diagnose gestellt ist, ist es ebenso wichtig, die richtige Behandlungsoption für jeden einzelnen Patienten zu wählen. Normalerweise wird dabei mit einer Tropfentherapie oder mit einer Laserbehandlung begonnen. Bei fortgeschrittenem oder fortschreitendem Glaukom bzw. einer Therapieunverträglichkeit muss möglicherweise eine Operation in Betracht gezogen werden.

Vieles hat sich geändert seit der Veröffentlichung der ersten großen Glaukomstudien vor über 20 Jahren. Die Ocular Hypertension Treatment Study (OHTS) bestimmte Risikofaktoren für das Glaukom und lieferte den ersten Beweis, dass die Senkung des Augeninnendrucks (IOD) das Auftreten des Glaukoms wirksam verzögerte oder ganz verhinderte [2, 3]. Obwohl die ursprüngliche Studie darauf ausgelegt war, Risikofaktoren für die Entwicklung des primären Offenwinkelglaukoms (POAG) zu untersuchen, lieferten viele nachfolgende Analysen mehr Details zu Art und Entwicklung der Krankheit. Selbst in den letzten Jahren sind immer noch neue Daten veröffentlicht worden $[4,5]$. Ähnlich wie bei OHTS wurden in der Erstbehandlungs-Glaukom-Studie (CITGS) zunächst Unterschiede im Verlauf von Glaukompatienten untersucht, die entweder mit Tropfen behandelt wurden oder direkt eine Operation erhielten. Immer noch werden Daten daraus produziert. Subanalysen wurden noch bis 2020 durchgeführt [6-9]. Studien zu Lasertherapien oder auch Operationen in der Behandlung des Glaukoms waren bislang eher begrenzt. Die Glaukom-Laser-Studie (GLT) und die Glaukom-Laser-Follow-up-Studie (GLTFS) waren die ersten Studien, die die Wirksamkeit der Lasertrabekuloplastik als Alternative zur Tropfenbehandlung zeigten [10, 11]. Die Advanced GlaukomInterventionsstudie (AGIS) untersuchte und verglich die Langzeitergebnisse nach Trabekulektomie und Argonlasertrabekuloplastik in Augen von Patienten, bei denen eine initiale medizinische Behandlung des Glaukoms fehlgeschlagen war [12]. 
In den letzten 10 Jahren haben wir eine Revolution in der Diagnose, Bildgebung und Behandlung von Glaukomen erlebt. Dies spiegelt die Fortschritte wider, die wir im Bereich des Verständnisses für die Erkrankung und bei der Entwicklung verschiedener und wirksamer Verfahren für die Behandlung erzielt haben. Obwohl ursprünglich als revolutionär angesehen, haben sich die meisten dieser Konzepte im Laufe der Jahre weiterentwickelt und werden jetzt durch starke Evidenz gestützt, die letztendlich immer unsere Entscheidungsfindung leiten sollte. Studien wie die britische Glaukombehandlungsstudie (UKGTS) und die automatisierte Glaukomtestbewertung (GATE) haben zuvor nicht untersuchte Aspekte des Managements des Behandlung und der Diagnosestellung beleuchtet [13-16]. Es wurden auch mehrere Schlüsselstudien durchgeführt, darunter LiGHT und ZAP, die sich speziell mit den Indikationen und der Wirksamkeit der Laserbehandlung bei primärem Offenwinkelglaukom sowie primärem Winkelblock befassen [17, 18]. Diese Studien haben uns mehr Beweise geliefert und Vertrauen in die Empfehlung einer Laserbehandlung für bestimmte Patienten gegeben [19]. Obwohl die primäre Behandlung des Glaukoms am häufigsten in der Gabe von Augentropfen oder Laser besteht, ist bei Patienten mit klinisch signifikantem Fortschreiten und unkontrollierter Erkrankung trotz maximaler medizinischer Therapie immer noch ein chirurgischer Eingriff erforderlich. Viele chirurgische Studien haben inzwischen Langzeitergebnisse veröffentlicht, die dem Glaukomspezialisten helfen, das richtige Gleichgewicht zwischen Risiko und Wirksamkeit zu finden, wenn er invasive Verfahren für seine Patienten in Betracht zieht. Große Studien wie die Tube Versus Trabeculectomy(TVT)- und die Primary Tube Versus Trabeculectomy(PTVT)Studie haben uns einen guten Einblick in die Erfolgsraten und Komplikationen der beiden weltweit durchgeführten Hauptglaukomverfahren gegeben [20-24]. Darüber hinaus haben die Studien Ahmed Baerveldt Comparison ( $A B C$ ) und Ahmed versus Baerveldt (AVB) jetzt 5-Jahres-Daten veröffentlicht, die auch einen Vergleich zwischen den verwendeten Glaukomdrainageimplantaten ermöglichen.
Während eine Vielzahl von Studien über verschiedene Arten von Glaukomverfahren einschließlich der sog. „minimal-invasiven Glaukomoperationen" berichtet hat, gibt es nun randomisierte Vergleiche, die sie mit dem Goldstandard „Trabekulektomie" vergleichen. Dieser ist als Referenzstandard für den Kliniker sehr wichtig, um die tatsächliche Wirksamkeit dieser Methoden zu beurteilen [25]. Eine weitere wichtige Studie zur Glaukomchirurgie bei fortgeschrittenen Erkrankungen (TAGS) wird eine wichtige Determinante dafür sein, wie wir Patienten behandeln, die sich mit neu diagnostiziertem fortgeschrittenem Glaukom vorstellen [26]. Ziel dieses Leitthemas ist es daher, die relevantesten Glaukomstudien zur Diagnose und Behandlung zusammenzufassen, die im letzten Jahrzehnt veröffentlicht wurden, um einen evidenzbasierten Ansatz für das Glaukommanagement zu liefern.

Korrespondenzadresse

Dr. Karl Mercieca, MD PGCMEd FRCOphth FEBOS-GL

Augenklinik, Universitätsklinikum Bonn Ernst-Abbe-Str. 2, 53127 Bonn, Deutschland Karl.Mercieca@ukbonn.de

Interessenkonflikt. K. Mercieca gibt an, dass kein Interessenkonflikt besteht.

\section{Literatur}

1. Tham YC, Li X, Wong TY, Quigley HA, Aung T, Cheng CY (2014) Global prevalence of glaucoma and projections of glaucoma burden through 2040: a systematic review and meta-analysis. Ophthalmology 121:2081-2090

2. Kass MA, Heuer DK, Higginbotham EJ, Johnson CA, Keltner JL, Miller JP, Parrish RK 2nd, Wilson MR, Gordon MO (2002) The Ocular Hypertension Treatment Study: a randomized trial determines that topical ocular hypotensive medication delays or prevents the onset of primary open-angle glaucoma. Arch Ophthalmol 120(6):701-713

3. Gordon MO, Beiser JA, Brandt JD, Heuer DK, Higginbotham EJ, Johnson CA, Keltner JL, Miller JP, Parrish RK 2nd, Wilson MR, Kass MA (2002) The Ocular Hypertension Treatment Study: baseline factors that predict the onset of primary openangleglaucoma. Arch Ophthalmol 120(6):714-720

4. Budenz DL, Huecker JB, Gedde SJ, Gordon M, Kass M, Ocular Hypertension Treatment Study Group. (2017) Thirteen-year follow-up of optic disc hemorrhages in the ocular hypertension treatment study. Am J Ophthalmol 174:126-133

5. Cousins X, Chou JC, Shen LQ, Gordon MO, Kass MA, Ritch R, Pasquale LR (2020) Densitometric profiles of optic disc hemorrhages in the ocular hypertension treatment study. Am J Ophthalmol 217:10-19

6. Musch DC, Gillespie BW, Lichter PR, Niziol LM, Janz NK, Investigators CS (2009) Visual field progression in the Collaborative Initial Glaucoma TreatmentStudy theimpact of treatmentand other baseline factors. Ophthalmology 116:200-207

7. Musch DC, Lichter PR, Guire KE, Standardi CL (1999) The Collaborative Initial Glaucoma Treatment Study: study design, methods, and baseline characteristics of enrolled patients. Ophthalmology 106(4):653-662

8. Feiner L, Piltz-Seymour JR (2003) Collaborative Initial Glaucoma Treatment Study:a summary of results to date. Curr Opin Ophthalmol 14(2):106-111

9. Newman-Casey PA, Niziol LM, Gillespie BW, Janz NK, Lichter PR, Musch DC (2020) The association between medication adherence and visual field progression in the collaborative initial glaucoma treatment study. Ophthalmology 127(4):477-483

10. Glaucoma Laser Trial Research Group (1990) The glaucoma laser trial (GLT): 2. Results of argon laser trabeculoplasty vs. topical medicines. Ophthalmology 97(11):1403-1413

11. Glaucoma Laser Trial Research Group (1995) The glaucoma laser trial (GLT) and glaucoma laser trial follow up study: 7. Results. Am J Ophthalmol 120(6):718-731

12. AGIS Investigators (1998) Advanced Glaucoma Intervention Study (AGIS): 4. comparison of treatment outcomes within race: $7 \mathrm{yr}$ results. Ophthalmology 105:1146-1164

13. Garway-Heath DF, Lascaratos G, Bunce C, Crabb DP, Russell RA, Shah A, United Kingdom Glaucoma Treatment Study Investigators (2013) The United Kingdom Glaucoma Treatment Study: a multicenter, randomized, placebo-controlled clinical trial: design and methodology. Ophthalmology 120(1):68-76

14. Jones L, Garway-Heath DF, Azuaro-Blanco A, Crabb DP (2019) Are patient self-reported outcome measures sensitive enough to be used as end points in clinical trials?: evidence from the United Kingdom glaucoma treatment study. Ophthalmology 126:682-689

15. Founti P, Bunce C, Khawaja AP, Doré CJ, MohamedNoriega J, Garway-Heath DF, United Kingdom Glaucoma Treatment Study Group (2020) Risk factors for visual field deterioration in the united kingdom glaucoma treatment study. Ophthalmology 127(12):1642-1651

16. Azuara-Blanco A, Banister K, Boachie C, MCMeekin P, Gray J, Burr J, Bourne R, GarwayHeath D, Batterbury M, Hernandez R, McPherson G, Ramsay C, Cook J (2016) Automated imaging technologies for the diagnosis of glaucoma: a comparative diagnostic study for the evaluation of the diagnostic accuracy, performance as triage tests and cost-effectiveness (GATE study). Health Technol Assess 20:8

17. Gazzard G, Konstantakopoulou E, Garway-Heath D, Garg A, Vickerstaff V, Hunter R, Ambler G, Bunce C, Wormald R, Nathwani N, Barton K, Rubin G, Buszewicz M, LiGHT Trial Study Group. (2019) Selective laser trabeculoplasty versus eye drops for first-line treatment of ocular hypertension and glaucoma (LiGHT): a multicentre randomised controlled trial. Lancet 393(10180):1505-1516

18. He M, Jiang Y, Huang S, Chang DS, Munoz B, Aung T, Foster PJ, Friedman DS (2019) Laser peripheral iridotomy for the prevention of angle closure: a single-centre, randomised controlled trial. Lancet 


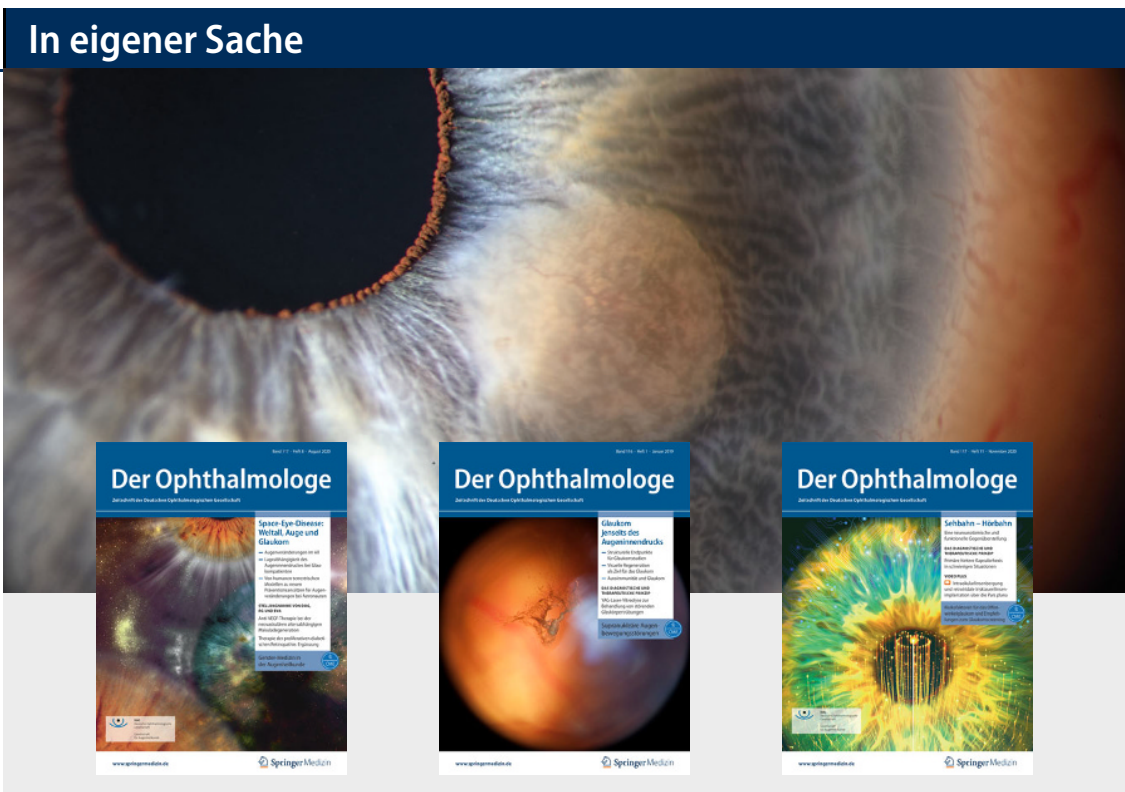

393(10181):1609-1618. https://doi.org/10.1016/ S0140-6736(18)32607-2

19. Khawaja AP, Campbell JH, Kirby N, Chandwani HS, Keyzor I, Parekh M, McNaught Al (2020) Realworld outcomes of selective laser Trabeculoplasty in the United Kingdom. UK glaucoma real-world data consortium. Ophthalmology 127(6):748-757

20. Gedde SJ, Schiffman JC, Feuer WJ, Parrish RK, Heuer DK, Brandt JD et al (2005) The tube versus trabeculectomy study: design and baseline characteristics of study patients. Am J Ophthalmol 140(2):275-287

21. Gedde SJ, Schiffman JC, Feuer WJ, Herndon LW Brandt JD, Budenz DL et al (2009) Three-year follow-up of the tube versus trabeculectomy study. Am JOphtalmol 148(5):670-684

22. Gedde SJ, Schiffman JC, Feuer WJ, Herndon LW Brandt JD, Budenz DL et al (2012) Treatment outcomes in the Tube Versus Trabeculectomy (TVT) study after five years of follow-up. Am J Opthalmol 153(5):789-803

23. Gedde SJ, Feuer WJ, Shi W, Ahmed IIK, Brandt J (2018) Treatment outcomes in the primary tube versus trabeculectomy study after 1 year of followup. Am JOphthalmol 125(5):650-663

24. Gedde SJ, Feuer WJ, Lim KS, Barton K, Goyal S, Ahmed IIK et al (2019) Treatment outcomes in the primary tube versus trabeculectomy study after 3 years of follow-up. Ophthalmology 127(3):333-345

25. A randomized study comparing the safety and efficacy of the InnFocus MicroShuntTM glaucoma drainage system to standard trabeculectomy in subjects with primary open angle glaucoma. ClinicalTrials.gov Identifier: NCT01881425

26. King AJ, Fernie G, Azuara-Blanco A, Burr JM, Garway-Heath D, Sparrow JM, Vale L, Hudson J, MacLennan G, McDonald A, Barton K, Norrie J (2017) Treatment of Advanced Glaucoma Study: a multicentre randomised controlled trial comparing primary medical treatment with primary trabeculectomy formpeople with newly diagnosed advanced glaucoma-study protocol. $\mathrm{Br} \mathrm{J}$ Ophthalmol 102(7):922-928

\section{Leitthemenübersicht von Der Ophthalmologe}

Der Ophthalmologe bietet Ihnen jeden Monat umfassende und aktuelle Beiträge zu interessanten Themenschwerpunkten aus allen Bereichen der Augenheilkunde.

Rückblick - 2020

01/20 Originalien

02/20 Periokuläres Basalzellkarzinom

03/20 Maximalinvasive Vorder-

abschnittschirurgie im Kindesalter

04/20 Qualitätssicherung bei der IVOM

05/20 Der Femtosekundenlaser in der Kataraktchirurgie

06/20 Personalisierte Medizin in der Behandlung von Augentumoren 07/20 SARS-CoV-2-Infektion und Auge 08/20 Space-Eye-Disease:Weltall, Auge und Glaukom

09/20 Retinologische Notfälle

10/20 Künstliche Intelligenz

11/20 Sehbahn - Hörbahn. Eine neuroanatomische und funktionelle Gegenüberstellung 12/20 Mikrochirurgische Operationstechniken aus ophthalmopathologischer Sicht

SpringerMedizin.de bietet Ihnen Zugang zu allen elektronisch verfügbaren Ausgaben Ihrer Zeitschrift - unabhängig davon, seit wann Sie die Zeitschrift abonniert haben.

\section{Vorschau - 2021}

01/21 Die Proliferative Vitreoretinopathie

02/21 Neuronale Ceroid-Lipofuszinosen

03/21 Die postoperative Endophthalmitis 04/21 OCT-Biomarker bei traktiven Makulopathien

05/21 Neue Glaukommedikamente

06/21 Hochrisikokeratoplastik

07/21 Originalien

08/21 Pseudotumor orbitae

09/21 Neue digitale Möglichkeiten für die Augenheilkunde

10/21 Orbitale Tumoren

11/21 Der akute retinale Zentralarterienverschluss

12/21 Schlüsselstudien zum Glaukom der letzten 10 Jahre

(Änderungen vorbehalten)

Alle Inhalte der Zeitschrift finden Sie unter www.springermedizin.de/ der-ophthalmologe 